\title{
Effects of Flux Creep in Bulk and Single Crystals of $\mathrm{Bi}_{2} \mathrm{Sr}_{2} \mathrm{CaCu}_{2} \mathrm{O}_{8+\delta}$ Superconductor
}

\author{
Mortady I. Youssif \\ Physics Department, Faculty of Science at Demiatta, \\ Mansoura University, New Demiatta 34517, Egypt
}

Low-field AC susceptibility studies of bulk and single crystals samples of $\mathrm{Bi}_{2} \mathrm{Sr}_{2} \mathrm{CaCu}_{2} \mathrm{O}_{8+\delta}(\mathrm{Bi}-2212)$ have been carried out in the frequency range up to $1 \mathrm{KHz}$. At constant AC field amplitude, the dissipative component of $A C$ susceptibility shifts to higher temperatures with increasing AC field frequency. Moreover, the thermal activation energy $\left(E_{a}\right)$ for flux creep in the crystals decreases with increasing AC field amplitude up to $800 \mathrm{Am}^{-1}$. These results are explained in terms of the effect of flux creep and Lorentz force $(J \times B)$ in high $T_{c^{-}}$ superconductors. 


\section{Introduction:}

The phenomenon of flux creep has received much attention in the case of high- $T_{c}$ oxide superconductors. In these materials, the grain boundaries are weak links in which the flux can creep at a relatively lower field than in the grains. Furthermore, because of the higher operating temperatures of the oxide superconductors, the thermal activation responsible for creep is easier in these materials as compared to the conventional superconductors. The activation energy for flux creep in bulk and single crystals of $\mathrm{YBa}_{2} \mathrm{Cu}_{3} \mathrm{O}_{7-\delta}(\mathrm{Y}-123)$ has been estimated by variety of techniques such as DC magnetization, AC susceptibility and resistivity under applied field [1-6]. The reported values of the activation energy vary widely depending upon the measurement technique and the microstructure features of the sample.

Low field AC susceptibility has been used to determine the flux creep activation energies in bulk Y-123 system [1,4]. Several attempts have been made to explain the observed increase of $T_{p}$, the temperature corresponding to the $\chi^{\prime \prime}$ peak, with the frequency of the AC field. Nikolo et al. [1] have been employed Anderson's flux creep model [7] and discussed the measured $\chi^{\prime \prime}$ peak shifts with frequency in terms of an activation energy for flux vortex depinning. A similar approach was used earlier by Malozemoff et al. [8] to interpret the $\chi^{\prime \prime}$ peak frequency dependence for $\mathrm{Y}-123$ and $\mathrm{Bi}_{2} \mathrm{Sr}_{2} \mathrm{CaCl}_{2} \mathrm{O}_{8+\delta}(\mathrm{Bi}-2212)$ single crystals in the presence of strong DC magnetic field. Müller [9] proposed a model to determine the temperature and AC field amplitude dependence of the complex susceptibility in granular superconductors. In a further extension of Müller's model [10] which incorporated Anderson's flux creep theory [7] it was possible to explain the observed increase of $T_{p}$ with the frequency of the AC field. It was further predicted that, for suitably higher magnetic fields, the smaller the grain size of the sample, the easier is the flux creep and stronger is the frequency dependence of $T_{p}$, which has also been confirmed experimentally [1,4,11-13].

\section{Experimental Details:}

Polycrystalline samples of $\mathrm{Bi}_{2} \mathrm{Sr}_{2} \mathrm{CaCu}_{2} \mathrm{O}_{8+\delta}(\mathrm{Bi}-2212)$ were prepared by a solid-state reaction between $\mathrm{Bi}_{2} \mathrm{O}_{3}, \mathrm{SrCO}_{3}, \mathrm{CaCO}_{3}$ and $\mathrm{CuO}$ powders. Appropriate amounts of the powders were mixed and calcined at $820^{\circ} \mathrm{C}$ for 12 $\mathrm{h}$ in air. The calcined powder was pulverized, cold-pressed into a disk shape, sintered at $860{ }^{\circ} \mathrm{C}$ for $24 \mathrm{~h}$ in air, and then furnace-cooled to room temperature. The single crystal samples of Bi-2212 were grown by the noval flux method. The crystals were having a plate-like shape with c-axis perpendicular to the surface. The sizes of the crystals were in the range $2 \times 3 \mathrm{~mm}^{2}$ to $3 \times 6 \mathrm{~mm}^{2}$. The absolute values of the real $\left(\chi^{\prime}\right)$ and the imaginary $\left(\chi^{\prime \prime}\right)$ components of the $\mathrm{AC}$ 
susceptibility were measured using low field AC susceptometer based on the mutual inductance method [14]. A copper-constantan thermocouple (type T) is used to measure the sample temperature and is connected to a temperature controller R/S (Cole-Parmer) which is designed to maintain a constant, pre-set process temperature $\left( \pm 0.1^{\circ} \mathrm{K}\right)$. The Bulk samples were placed in the holder and the AC field amplitudes of $0.8,8$ and $80 \mathrm{Am}^{-1}$ were applied parallel to the longest dimension of the samples. To investigate the effect of flux creep in single crystals, the magnetic field was applied parallel and perpendicular to the c-axis of the crystals, respectively. For $\mathrm{H} / /$ to the c-axis, the $\mathrm{AC}$ field amplitudes were 8, 240, $600 \mathrm{Am}^{-1}$, and were $240,600,800 \mathrm{Am}^{-1}$ for $\mathrm{H} \perp$ to the c-axis. All the measurements were made in the temperatures between $77^{\circ} \mathrm{K}$ and $90{ }^{\circ} \mathrm{K}$ at different $\mathrm{AC}$ field frequencies of $111.1,333.3$ and $1000 \mathrm{~Hz}$, respectively. From real and imaginary components versus temperature plots, the values of the transition temperature $T_{c}\left(\chi^{\prime}\right)$ and the peak temperature $T_{p}$ (corresponding to peak $\chi^{\prime \prime}$ ) were estimated.

\section{Results and Discussion:}

Figures 1 and 2 show the real and imaginary parts of $\mathrm{AC}$ magnetic susceptibility for $\mathrm{Bi}_{2} \mathrm{Sr}_{2} \mathrm{CaCu}_{2} \mathrm{O}_{8+\delta}$ single crystal as a function of temperature with various AC field frequencies at AC field amplitude of $240 \mathrm{Am}^{-1}$ with the magnetic field applied parallel and perpendicular to the c-axis of the crystal, respectively. From the $\chi^{\prime}$ component of the AC susceptibility the diamagnetic onset is found to be at $\sim 86.75^{\circ} \mathrm{K}$ for single crystal and at $\sim 87.0^{\circ} \mathrm{K}$ for polycrystalline samples, respectively.

Nikolo and Goldfarb [1] defined the activation energy for flux creep $\left(E_{a}\right)$ in terms of the AC field frequency $f$ and the $\chi^{\prime \prime}$ peak temperature $T_{p}$. The expression for $E_{a}$ is given by:

$$
f=f_{0} \exp \left[-E_{a} / K_{B} T_{p}\right]
$$

where $K_{B}$ is the Boltzmann's constant and $f_{0}$ is a constant with dimension of frequency. By taking the logarithmic of the two sides of Eq. (1), we fined that;

$$
\ln f=\ln f_{0}-E_{a} / K_{B} T_{P}
$$

Using Eq. (2), the activation energy $E_{a}$ can be estimated by the slope of the $\ln (f)$ versus $1 / T_{p}$ plots, which are straight lines. 
The variation of $\chi$ with temperatures in Figs. ( $1 \& 2)$ shows a small shift in the susceptibility curves to higher temperatures $\left(T_{p}\right)$ with increasing the field frequency, at constant $\mathrm{AC}$ field amplitude. This shift in the AC susceptibility curves is arising from the effect of flux creep in the crystal [10]. Since, the AC field inside the superconductor at $T_{p}$ just fully penetrates the crystals when the AC field amplitude reaches its maximum or minimum. Therefore, with increasing the AC field frequency, the vortices have less and less time to creep into the superconductor during the AC field cycle. In order to obtain full flux penetration, a weaker pinning force density is needed to compensate for less efficient creep. As the pinning force density weakens with increasing temperature, the peak temperature $T_{p}$, must increases with increasing AC field frequency. Tables ( 1 and 2 ) summarizes the values of $T_{c}$ (diamagnetic onset), the $\chi^{\prime \prime}$ peak temperature $T_{p}$, and the estimated values of $E_{a}$ (the activation energy for flux creep) as a function of $\mathrm{AC}$ field amplitudes at various frequencies for single crystals of $\mathrm{Bi}_{2} \mathrm{Sr}_{2} \mathrm{CaCu}_{2} \mathrm{O}_{8+\delta}$ with $\mathrm{H} / /$ to the c-axis and $\mathrm{H} \perp$ to the c-axis of the crystals, respectively. For bulk samples of Bi-2212, the parameters $T_{c}, T_{p}$, and $E_{a}$ as a function of AC field amplitudes at frequency $f=$ 111.1 Hz are listed in Table (3).

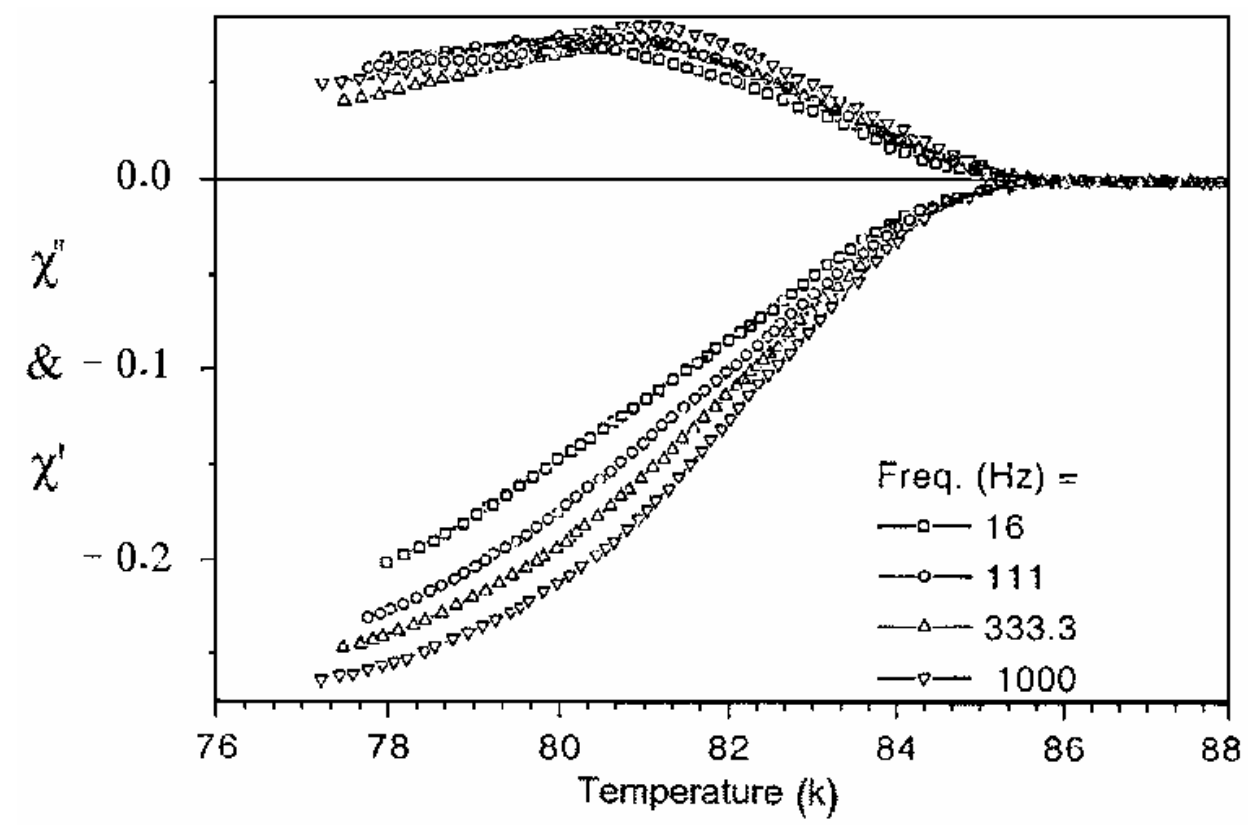

Fig. (1): $\mathrm{AC}$ magnetic susceptibility as a function of temperature with various $\mathrm{AC}$ field frequencies for single crystals of $\mathrm{Bi}_{2} \mathrm{Sr}_{2} \mathrm{CaCu}_{2} \mathrm{O}_{8+\delta}$ at $\mathrm{AC}$ field amplitude of $240 \mathrm{Am}^{-1}$ with $\mathrm{H} / /$ to c-axis of the crystal. 


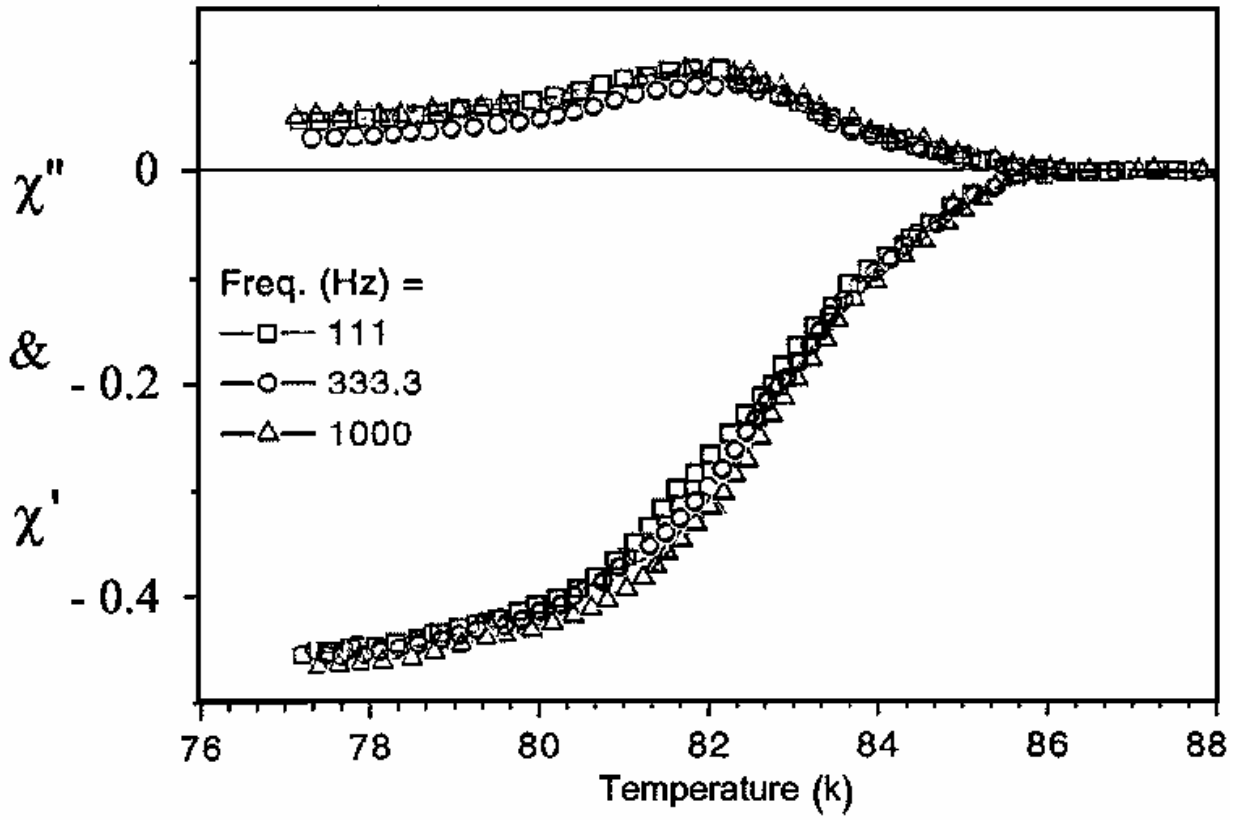

Fig. (2): $\mathrm{AC}$ magnetic susceptibility as a function of temperature with various $\mathrm{AC}$ field frequencies for single crystals of $\mathrm{Bi}_{2} \mathrm{Sr}_{2} \mathrm{CaCl}_{2} \mathrm{O}_{8+\delta}$ at $\mathrm{AC}$ field amplitude of $240 \mathrm{Am}^{-1}$ with $\mathrm{H} \perp$ to $\mathrm{c}$-axis of the crystal.

Table (1). The parameters $T_{p}$, estimated values of $E_{a}$ (the activation energy for flux creep), $T_{c}$ (diamagnetic onset), zero-temperature pinning potential $\beta I_{o}(0)$, and grain sizes $R_{g}$ as a function of $\mathrm{AC}$ field amplitudes at various frequencies for single crystals of $\mathrm{Bi}_{2} \mathrm{Sr}_{2} \mathrm{CaCu}_{2} \mathrm{O}_{8+\delta}$ with $\mathrm{H} / /$ to the c-axis of the crystal.

\begin{tabular}{|c|c|c|c|c|c|c|}
\hline$H_{a}(\mathrm{~A} / \mathrm{m})$ & $f \quad(\mathrm{~Hz})$ & $T_{p}(\mathrm{~K})$ & $\begin{array}{c}\text { Estimated } \\
\text { values of } E_{a} \\
(\mathrm{eV})\end{array}$ & $T_{c}(\mathrm{~K})$ & $\begin{array}{c}\beta I_{o}(0) \times 10^{-4} \\
(\text { Joule/Web. })\end{array}$ & $\begin{array}{c}\text { Estimated } \\
R_{g} \times 10^{-6}(\mathrm{~m})\end{array}$ \\
\hline 8.00 & 111.1 & 83.15 & 2.360 & 86.75 & 11.286 & 0.887 \\
& 333.3 & 83.49 & & & & \\
& 1000.0 & 83.41 & & & & \\
\hline \multirow{3}{*}{240} & 111.1 & 80.49 & 1.734 & & & \\
& 333.3 & 80.84 & & & & \\
& 1000.0 & 81.18 & & & & \\
\hline \multirow{3}{*}{600} & 111.1 & 78.90 & 1.190 & & & \\
& 333.3 & 79.38 & & & & \\
& 1000.0 & 79.85 & & & & \\
\hline
\end{tabular}


Table (2). The parameters $T_{p}$, estimated values of $E_{a}, T_{c}$, the zero-temperature pinning potential $\beta I_{o}(0)$, and $R_{g}$ as a function of $\mathrm{AC}$ field amplitudes at various frequencies for single crystals of $\mathrm{Bi}_{2} \mathrm{Sr}_{2} \mathrm{CaCu}_{2} \mathrm{O}_{8+\delta}$ with $\mathrm{H} \perp$ to the c-axis of the crystal.

\begin{tabular}{|c|c|c|c|c|c|c|}
\hline$H_{a}(\mathrm{~A} / \mathrm{m})$ & $f(\mathrm{~Hz})$ & $T_{p}(\mathrm{~K})$ & $\begin{array}{c}\text { Estimated } \\
\text { values of } E_{a} \\
(\mathrm{eV})\end{array}$ & $T_{c}(\mathrm{~K})$ & $\begin{array}{c}\beta I_{o}(0) \times 10^{-4} \\
(\text { Joule/Web. })\end{array}$ & $\begin{array}{c}\text { Estimated } \\
R_{g} \times 10^{-6}(\mathrm{~m})\end{array}$ \\
\hline 240 & 111.1 & 81.75 & 1.807 & 86.75 & 9.708 & 0.668 \\
& 333.3 & 82.04 & & & & \\
& 1000.0 & 82.30 & & & & \\
\hline \multirow{3}{*}{600} & 111.1 & 80.50 & 1.356 & & & \\
& 333.3 & 81.00 & & & & \\
& 1000.0 & 81.45 & & & & \\
\hline \multirow{3}{*}{800} & 111.1 & 80.05 & 1.219 & & & \\
& 333.3 & 80.50 & & & & \\
& 1000.0 & 81.07 & & & & \\
\hline
\end{tabular}

Table (3). Values of $T_{p}, E_{a}, T_{c}, \beta I_{o}(0)$ and grain sizes $R_{g}$ parameters as a function of AC field amplitudes at frequency $f=111.1 \mathrm{~Hz}$ for $\mathrm{Bi}_{2} \mathrm{Sr}_{2} \mathrm{CaCu}_{2} \mathrm{O}_{8+\delta}$ bulk superconductors.

\begin{tabular}{|c|c|c|c|c|c|}
\hline$H_{a}(\mathrm{~A} / \mathrm{m})$ & $T_{p}(\mathrm{~K})$ & $\begin{array}{c}\text { Estimated values } \\
\text { of } E_{a}(\mathrm{eV})\end{array}$ & $T_{c}(\mathrm{~K})$ & $\begin{array}{c}\beta I_{o}(0) \times 10^{-4} \\
(\text { Joule } / \text { Web. })\end{array}$ & $\begin{array}{c}\text { Estimated } \\
R_{g} \times 10^{-6}(\mathrm{~m})\end{array}$ \\
\hline 0.8 & 85.90 & 0.500 & 87.0 & 1.942 & 1.367 \\
\hline 8.0 & 83.40 & 0.250 & & & \\
\hline 80 & 83.00 & 0.025 & & & \\
\hline
\end{tabular}

In Figs. (3 and 4), the variation of $\ln f$ versus $1 / T_{p}$ at different values of AC field amplitudes with the magnetic field applied parallel and perpendicular to the c-axis of the crystal are shown respectively. From the slope of these straight lines, the values of the activation energies at different fields were estimated.

According to Müller's critical state model [10] the shift in $T_{p}$ with AC field frequency indicates flux creep. In a further extension of this model, Kumaraswamy et al. [11] have neglected the low values of AC field amplitudes $\left(H_{a}\right)$ as compared to the field at the $\chi^{\prime \prime}$ peak $H_{o p}$. Based on this approach, he has derived an expression for the flux creep activation energies $\left(E_{a}\right)$ which is simply given by:

$$
E_{a}=\frac{\beta \mathrm{I}_{0}(0) \Phi_{0}}{2 \pi}-\frac{4 H_{a} \bar{R}_{g}{ }^{2} \Phi_{0}}{D}
$$




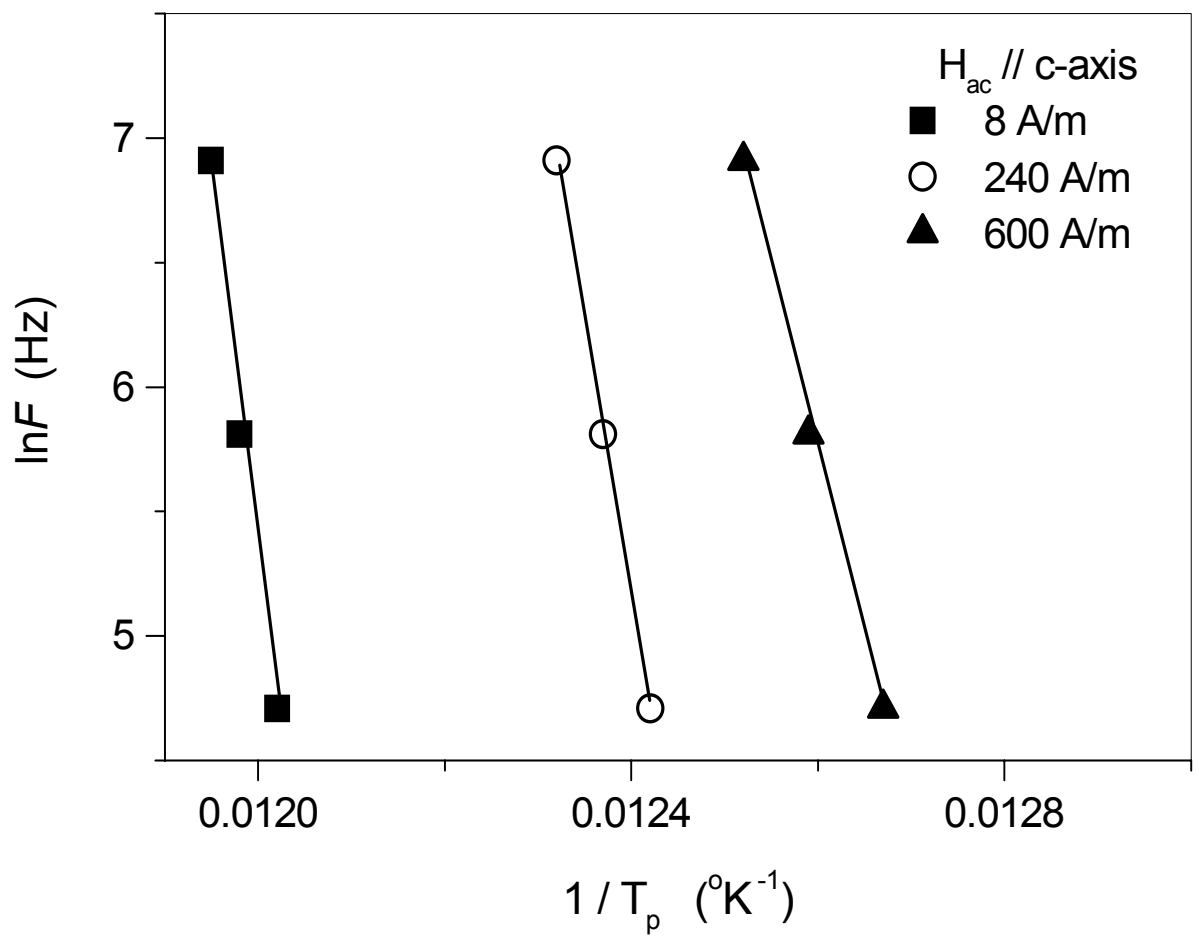

Fig. (3): Variation of $\ln (F)$ versus $1 / T_{p}$ at different values of $A C$ field amplitudes for single crystals of $\mathrm{Bi}_{2} \mathrm{Sr}_{2} \mathrm{CaCu}_{2} \mathrm{O}_{8+\delta}$ with $\mathrm{H} / /$ to c-axis of the crystal.

where $\Phi_{0}$ is the flux quantum $\left(\Phi_{0}=2.07 \times 10^{-15}\right.$ weber $), \beta I_{0}(0)$ is the zerotemperature pinning potential of the sample, $\bar{R}_{g}$ is the average grain size of the sample, and $D$ is the sample thickness (or the diameter in the case of cylindrical sample). By applying Eq. (3), the average grain size $\bar{R}_{g}$ can be estimated by the slope of the $E_{a}$ versus $H_{a}$ plots, which are straight lines. The pinning potential $\beta I_{0}(0)$ at zero Kelvin is calculated from the bisected part of $E_{a}$. Figure (5) shows the behavior of the activation energy as a function of the AC field amplitudes for bulk and single crystal samples of $\mathrm{Bi}_{2} \mathrm{Sr}_{2} \mathrm{CaCu}_{2} \mathrm{O}_{8+\delta}$ with $H_{a}$ parallel and perpendicular to the c-axis of the crystal, respectively. This figure shows clearly that the activation energy $E_{a}$ decreases with increasing AC field amplitude, $H_{a}$. This could be interpreted based on the fact that, with increasing AC field amplitude, the driving Lorentz force increase, and more flux can creep during the AC field cycle. From this figure together with Eq. (3), values of the average grain sizes and the zero-temperature pinning potential of single crystals and polycrystalline samples of $\mathrm{Bi}_{2} \mathrm{Sr}_{2} \mathrm{CaCu}_{2} \mathrm{O}_{8+\delta}$ have been calculated and listed in Tables 1,2, and 3, respectively. 


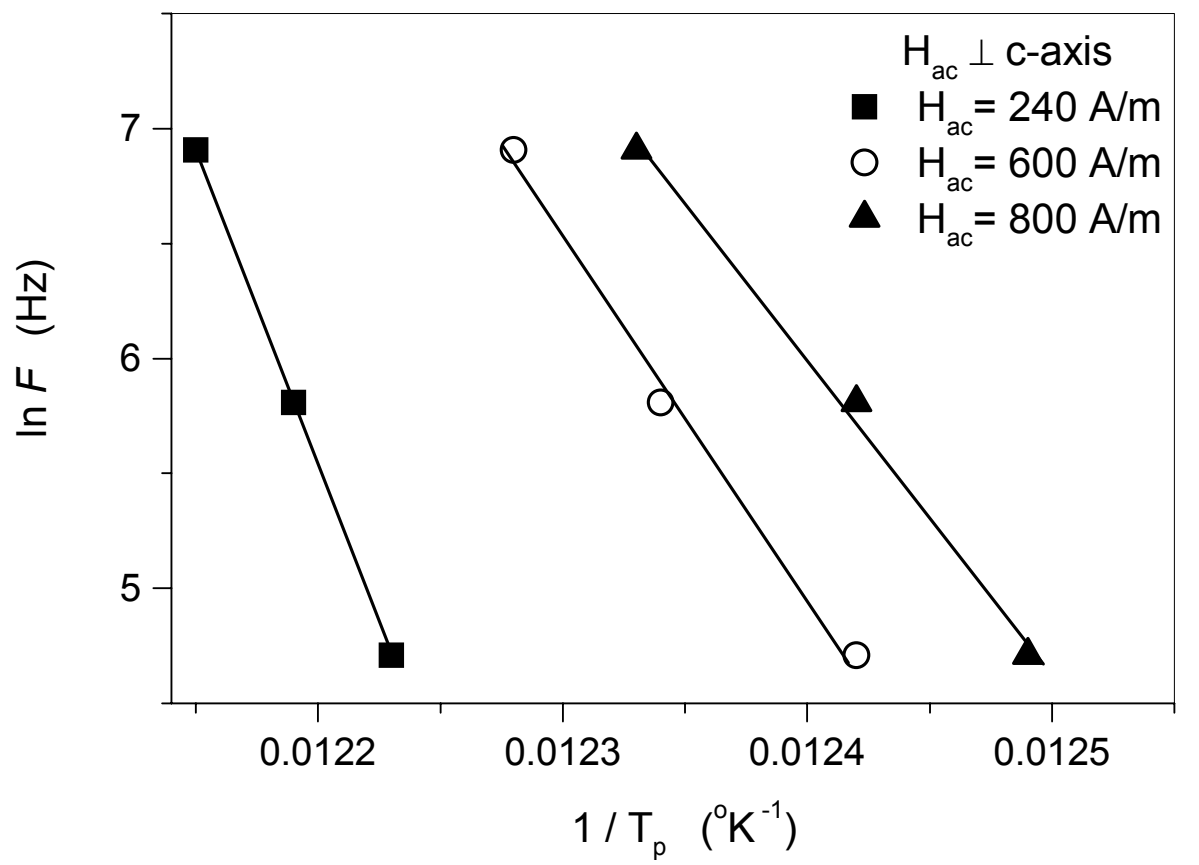

Fig. (4): Variation of $\ln (F)$ versus $1 / T_{p}$ at different values of $A C$ field amplitudes for single crystals of $\mathrm{Bi}_{2} \mathrm{Sr}_{2} \mathrm{CaCu}_{2} \mathrm{O}_{8+\delta}$ with $\mathrm{H} \perp$ to c-axis of the crystal.

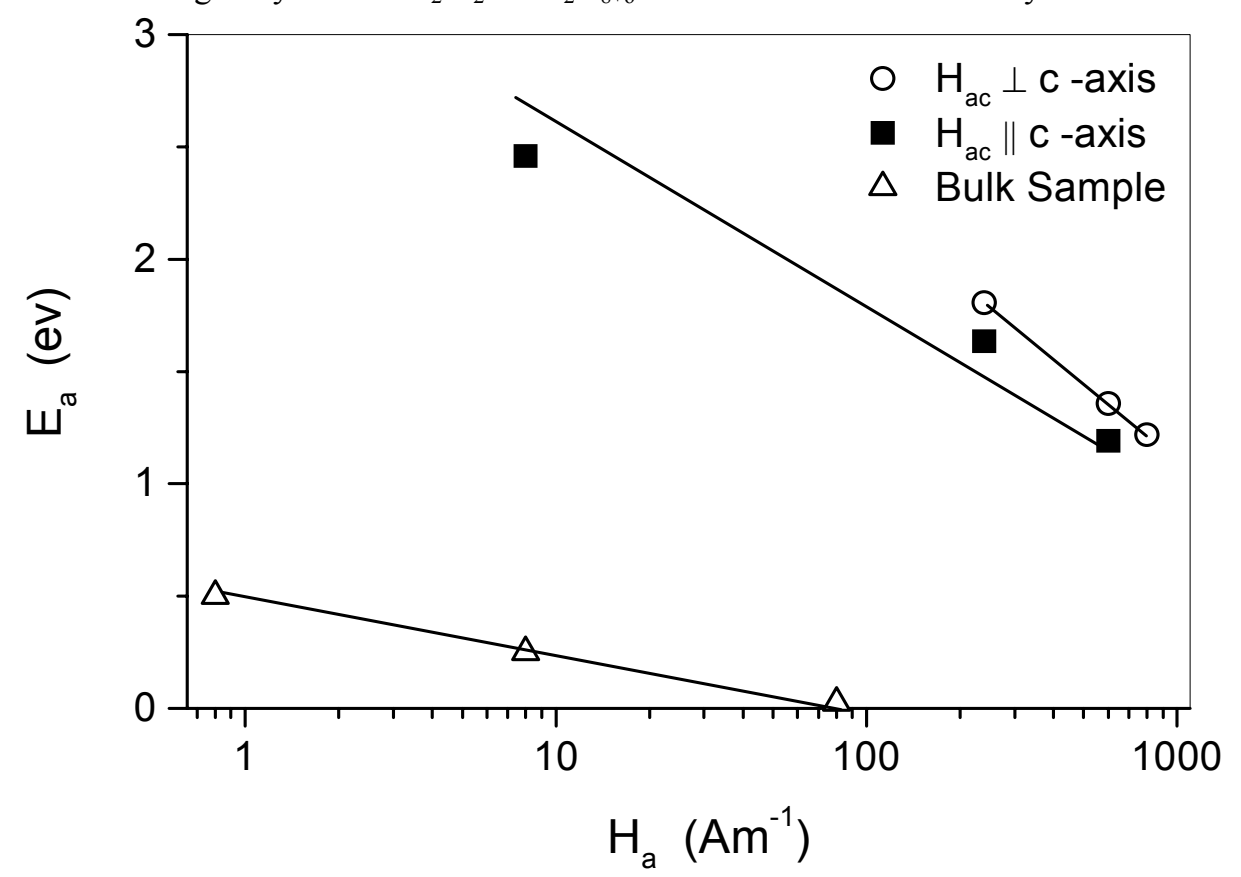

Fig. (5): The activation energy $(E a)$ versus $\mathrm{AC}$ field amplitudes for bulk and single crystals of $\mathrm{Bi}_{2} \mathrm{Sr}_{2} \mathrm{CaCu}_{2} \mathrm{O}_{8+\delta}$ with $\mathrm{H} / /$ to the c-axis and $\mathrm{H} \perp$ to the $\mathrm{c}$-axis of the crystals. 


\section{Conclusions:}

The phenomenon of flux creep should be considered for bulk and single crystals of $\mathrm{Bi}_{2} \mathrm{Sr}_{2} \mathrm{CaCu}_{2} \mathrm{O}_{8+\delta}(\mathrm{Bi}-2212)$ superconductor system. At constant low AC field amplitude, the dissipative component of AC susceptibility shifts to higher temperatures with increasing $\mathrm{AC}$ field frequency. We have interpreted this shift in terms of flux creep in high- $T_{c}$ superconductors. With increasing AC field amplitude, the Lorentz force $(J \times B)$ increases and a decrease in the thermal activation energy $\left(E_{a}\right)$ was observed.

\section{References:}

1. M. Nikolo and R.B. Goldfarb, Phys. Rev. B, 39, 6615 (1989).

2. M.P. Maley, J.O. Willis, Phys. Rev. B, 42, 2639 (1990).

3. M.D. Lan, J.Z. Liu, R.N. Shelton, Phys. Rev. B, 44, 2751 (1991).

4. S. Lofland, M.X. Huang, S.M. Bhagat, Physica C, 203, 271 (1992).

5. C. Varanasi, P.J. McGinn, H.A. Blackstead, D.B. Pulling, Appl. Phys. Lett., 67, 1004 (1995).

6. D. Berling, D. Bolmont, B. Logel, A. Mehdaoui, Solid State Commun., 97, 731 (1996).

7. P.W. Anderson, Phys. Rev. Lett., 9, 309 (1962).

8. A.P. Malozemoff, T.K. Worthington, Y. Yeshurun, F. Holzberg, P.H. Kes, Phys. Rev. B, 38, 7203 (1988).

9. K.H. Müller, Physica C, 159, 717 (1989).

10. K.H. Müller, Physica C, 168, 585 (1990).

11. B.V. Kumaraswamy, Ratan Lal, A.V. Narlikar, Phys. Rev. B, 52, 1320 (1995).

12. Ph. Vanderbemden, Ch. Destombes, R. Cloots, M. Ausloos, Supercon. Sci. Technol., 11, 94 (1998).

13. A. Sedky, M.I. Youssif, J. Magn. Magn. Mater., 237, 22 (2001).

14. M.I. Youssif, A.A. Bahgat, I.A. Ali, Egypt. J. Sol., 23, 231 (2000). 